the editorial base alike. It is therefore important that the reviews that are eventually published provide the most informative answers to the most relevant questions to guide decision-making. Asking vague questions leads to vague answers and is a waste of time, money and resources.

Methods Any question arising from practice that has to do with choosing a suitable intervention for a particular health issue can be formulated to contain the elements $\mathrm{p}=$ Participants, $\mathrm{I}=$ Intervention(s), $\mathrm{C}=\mathrm{Control}$ and $\mathrm{O}=$ Outcome(s). Similarly, a research study evaluating the effectiveness of a particular intervention and a systematic review aiming to make a summary of all sufficiently similar studies ought to use this recipe. This talk will explore how each of these elements influence the whole review process from searching studies to making a synthesis of their findings and reporting results.

Result We will compare a convenience sample of five recent Cochrane Work reviews with another five recent nonCochrane reviews for their use of PICO and how it is implemented throughout the review.

Discussion PICO is a simple tool that will ensure that research will answer the question of interest that has arisen from practice. Ignoring PICO almost certainly leads to a biassed review process and consequently biassed review results. PICO is the most important ingredient in enabling evidence-based medicine.

\section{0b GRADE APPLIED IN A RECENT UPDATED COCHRANE REVIEW}

${ }^{1} \mathrm{HF}$ van der Molen, ${ }^{2} \mathrm{P}$ Basnet, ${ }^{2} \mathrm{HH}$ Verbeek. ${ }^{1}$ Academic Medical Centre, University of Amsterdam, Department: Coronel Institute of Occupational Health, Amsterdam Public Health research institute, Amsterdam, The Netherlands; ${ }^{2}$ Cochrane Work Review Group, Finnish Institute of Occupational Health, Kuopio, Finland

\subsection{6/oemed-2018-ICOHabstracts.360}

Evaluation of interventions to reduce occupational injuries in the construction industry are relatively scarce. Various interventions to prevent occupational injuries have been proposed and studied. In a Cochrane review we systematically summarise the most current scientific evidence on the effectiveness of interventions to prevent injuries associated with construction work. Most of these studies are analysed with an interrupted time series design, which are characterised by a higher risk of bias.

We use the GRADE (Grades of Recommendations, Assessment, Development and Evaluation) approach that systematically represents the factors important in interpretating evidence and results in a current update of our review. While the evidence can be different for each outcome, GRADE considers the evidence for each outcome and takes into account the magnitude of effect and ensures the process is systematic and transparent.

Rating of the evidence was done as follows: with RCTs we started at high quality and with observational studies we started at low quality. Then we downgraded if one of the following criteria were met: study limitations, inconsistency, indirectness, imprecision and publication bias. We upgraded observational studies if there have been dose-response, large effect size or an opposite effect of confounding. We constructed tables for every comparison for our interventions and our two primary outcomes fatal and non-fatal injuries because these were our inclusion criteria for the studies.

Applying GRADE and the difference with strength of association will be discussed based on the above mentioned update of our review. Also the differences in clarity of the conclusions with and without GRADE will be discussed.

\section{C UNEXPECTED INVITATION TO BECOME A CO-AUTHOR OR: HOW I LEARNED TO STOP WORRYING AND LOVE SYSTEMATIC REVIEWS}

TC Morata. National Institute for Occupational Safety and Health, Cincinnati, USA

10.1136/oemed-2018-ICOHabstracts.361

All of us are recipients of health care, and most of the ICOH Congress participants also provide health services. As patients and providers we expect that the service being rendered stands on a solid scientific base. Nowadays, with the expansion of publications and communication channels, we hear a lot about evidence-based practice (EBP) and systematic reviews. Systematic reviews, are the most important type of scientific review because they are central to evidence-based practice, but they can be misunderstood or even intimidating to some. Examination of the contributions of systematic reviews to occupational health and the processes to get familiarised, became a user and participate in the implementation of evidence-based practices to prevent work-related disorders. Locating pertinent Cochrane resources and reviews, and defining what are answerable questions and eligible sources of evidence for a Cochrane Review. Examples will focus on a Cochrane review that examined the effectiveness of enforcement tools for preventing occupational diseases and injuries and a second one that examined interventions to promote the use of hearing protections and other efforts to control noise and promote hearing loss prevention. To be able to offer evidence-based practices, occupational health professional need to recognise the need and approaches that will allow him/her to be a lifelong learner, by keeping current with evidence-based professional practice, and engage in continuing competence and professional development activities.

Disclaimer The findings and conclusions in this abstract have not been formally disseminated by the National Institute for Occupational Safety and Health and should not be construed to represent any agency determination or policy.

\section{$1710 \mathrm{~d}$ SYSTEMATIC REVIEWS AND EVIDENCE-BASED GUIDELINES, TWO OF A DIFFERENT KIND?}

Carel T Hulshof. Netherlands Society of Occupational Medicine (NVAB), Centre of Excellence, Utrecht, the Netherlands; Academic Medical Centre, dept. Coronel Institute of Occupational Health, Amsterdam, the Netherlands

\subsection{6/oemed-2018-ICOHabstracts.362}

In improving the quality and professional independence of Occupational Safety and Health (OSH) professionals, the development of an evidence-based practice plays a pivotal role. OSH professionals should strive to use scientific evidence as much as possible to support their decisions in daily practice and policy. However, in many situations, still a gap between evidence from research and decision-making in daily practice 
occurs. In attempts to bridge this gap, high-quality, relevant and up-to-date systematic reviews and evidence-based guidelines are both important tools. The processing of a systematic review and the development of an evidence-based guideline show a lot of similarities in undertaking research syntheses, developing methods for identifying, appraising, and synthesising evidence, and in translating research evidence into practice and policy. International organisations like Cochrane Collaboration and Guidelines International Network (G-I-N) collaborate in the development and adoption of tools like GRADE to rate the quality of evidence and to grade the strength of recommendations.

However, also differences can be identified. While systematic reviews investigate and summarise the scientific literature on effects of exposures, interventions, or diagnostic procedures in a systematic and methodologically rigorous way, it is clear that decision making in the field of OSH will seldom be based on research evidence alone but will incorporate professional expertise of professionals, ethical considerations, preferences and values of workers, and the policies of governments, companies and other stakeholders. Guidelines are defined as 'documents with recommendations to assist healthcare practitioners and healthcare users, intended to optimise quality of care, based on a systematic review of evidence and an assessment of the benefits and harms of the various care options, supplemented with expertise and experiences of both practitioners and users'. In particular when high quality evidence is not available, contradictory, or inappropriate, experiential and contextual knowledge may help guideline makers to formulate sensible recommendations.

\section{$1710 \mathrm{e}$ INTERVENTIONS TO INCREASE THE REPORTING OF OCCUPATIONAL DISEASES BY PHYSICIANS}

${ }^{1} S$ Curti, ${ }^{2} R$ Sauni* ${ }^{3} D$ Spreeuwers, ${ }^{4} A$ de Schryver, ${ }^{5} \mathrm{M}$ Valenty, ${ }^{6} S$ Rivière, ${ }^{1} S$ Mattioli. ${ }^{1}$ Department of Medical and Surgical Sciences, University of Bologna, Bologna, Italy; ${ }^{2}$ Department for Occupational Safety and Health, Ministry of Social Affairs and Health, Finland; ${ }^{3}$ Free University Medical Centre, Amsterdam, Netherlands; ${ }^{4}$ Epidemiology and Social Medicine, University of Antwerp, Antwerpen, Belgium; ${ }^{5}$ Département Santé Travail, Institut de Veille Sanitaire, Saint Maurice, France

\subsection{6/oemed-2018-ICOHabstracts.363}

Introduction Under-reporting of occupational diseases is an important issue worldwide. The collection of reliable data is essential for prevention programmes. Little is known about the effects of interventions for increasing the reporting of occupational diseases.

Methods We searched the Cochrane Occupational Safety and Health Group Specialised Register, the Cochrane Central Register of Controlled Trials (CENTRAL), MEDLINE (PubMed), EMBASE, OSH UPDATE, Database of Abstracts of Reviews of Effects (DARE), Open-SIGLE, and Health Evidence until January 2015. We also checked reference lists of relevant articles and contacted study authors.

Results We included 12 studies. Six studies evaluated the effectiveness of educational materials alone; one study evaluated the effectiveness of educational meetings; and four studies evaluated a combination of the two in increasing the reporting of occupational diseases by physicians. A further study evaluated the effectiveness of a complex educational campaign acting at society level. We found that the use of educational materials did not considerably increase the number of physicians reporting occupational diseases, but a legal obligation reminder message did. Furthermore, we found that the use of educational materials did not considerably increase the rate of reporting occupational diseases. Similarly, we found that the use of both educational materials and meetings did not considerably increase the number of physicians reporting occupational diseases or the rate of reporting. The same holds for the use of educational meetings alone. The use of an educational campaign appeared to increase the number of physicians reporting occupational diseases, although this was based on very low-quality evidence.

Discussion The studies provide evidence that educational materials, educational meetings, or a combination of the two do not considerably increase the reporting of occupational diseases. The use of a reminder message on the legal obligation to report might provide some positive results. We need highquality RCTs to corroborate these findings.

\section{$1710 f$ EMPTY REVIEWS: HOW, WHY OR WHY NOT?}

D FitzGerald. Medmark Occupational Healthcare, Cork, Ireland

\subsection{6/oemed-2018-ICOHabstracts.364}

Introduction Where no studies relevant to the scientific question being examined meet the pre-specified inclusion criteria, a systematic review becomes an 'empty review'. As such, no robust evidence based conclusions can be drawn from the findings of these reviews. It may be that such reviews have been undertaken where there is not yet a sufficient body of knowledge to allow for a systematic review being undertaken. In such circumstances, the publication of an empty systematic review may prompt the funding for and development of studies to answer the relevant scientific question. Also, in is much as there is a benefit in knowing what evidence based recommendations can be made in clinical practice, it is also useful to know what commonly used treatments are prescribed based on generally accepted standard practice rather than proven clinical benefit. It may also be, however, that the question being examined is too narrow or too focused, with excessively broad exclusion criteria, to be of relevance in a typical clinical setting. The development of such systematic reviews may be regarded as wasteful in resource limited circumstances.

\section{OVERVIEW OF EPICOH SPECIAL SESSION FOR ICOH 2018 - HIGHLIGHTED ISSUES IN OCCUPATIONAL EPIDEMIOLOGY}

Leslie London. School of Public Health and Family Medicine, University of Cape Town, South Africa

10.1136/oemed-2018-ICOHabstracts.365

Professors: ${ }^{1}$ Leon Guo, ${ }^{2}$ Po-Chin $\mathrm{Chu},{ }^{3} \mathrm{R}$ Mendes, ${ }^{4} \mathrm{O}$ Dumas, ${ }^{5}$ AM Neophytou

${ }^{1}$ National Institute of Environmental Health Sciences, National Health Research Institutes, Taiwan

${ }^{2}$ Environmental and Occupational Medicine, National Taiwan University College of Medicine and NTU Hospital, Taipei, Taiwan

${ }^{3}$ National Association of Occupational Medicine (ANAMT), São Paulo - SP, Brazil 